\title{
Neutral Volatile Components of Burley Tobacco*
}

\author{
by Takane Fujimori, Reiko Kasuga, Hajime Kaneko and Masao Noguchi \\ Central Research Institute, The Japan Tobacco and Salt Public Corporation, \\ Yokobama, Japan
}

\section{INTRODUCTION}

Tobacco aroma consists of a large number of minor components. $U_{p}$ to the present, more than 300 volatile compounds are known to exist in tobacco leaves. Most of these compounds are thought to be generated by the oxidative degradation of terpenoids such as carotenoids, thunberganoids, labdanoids and acyclic polyisoprenoids during the curing and aging process.

To find the representative aroma constituents of tobacco, we have been studying the essential oils of good quality Burley tobacco leaves. Burley tobacco represents about $10 \%$ of the tobacco grown in the world. It is an aircured type tobacco and possesses excellent smoking characteristics for American blend cigarette tobaccos.

\section{EXPERIMENTAL}

The process of extraction and fractionation of the essential oils of Burley tobacco is shown in Figure 1. The medium-range boiling point fraction (M-fraction) was obtained from $370 \mathrm{~kg}$ of air-cured U.S. Burley tobacco leaves by means of dichloromethane extraction, steam-

\footnotetext{
* Presented at the 7th International Congress of Essential Oils, Kyoto, Japan, in 1977 and, in part, at the 6th International Tobacco Scientific Congress (Coresta) held in Tokyo, Japan, in 1976.
}

distillation under reduced pressure, fractionation and distillation under reduced pressure. The M-fraction had a representative aroma of the essential oils. The column chromatography of the M-fraction was carried out using hexane and ether by stepwise increasing the polarity. The results of the chromatography are shown in Figure 2. The fractions obtained from the column chromatography were further separated by preparative gas chromatography. The materials isolated were analyzed by obtaining their MS, IR, NMR and UV spectra $(1,2)$.

Figure 2. Column chromatography of the medlum-range boiling point fraction (M-fractlon).

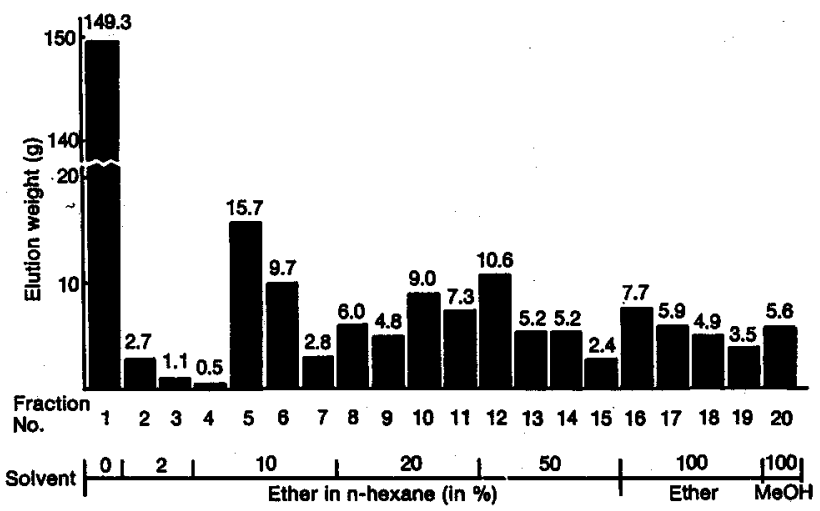


Figure 1. Process of extraction and fractionation.

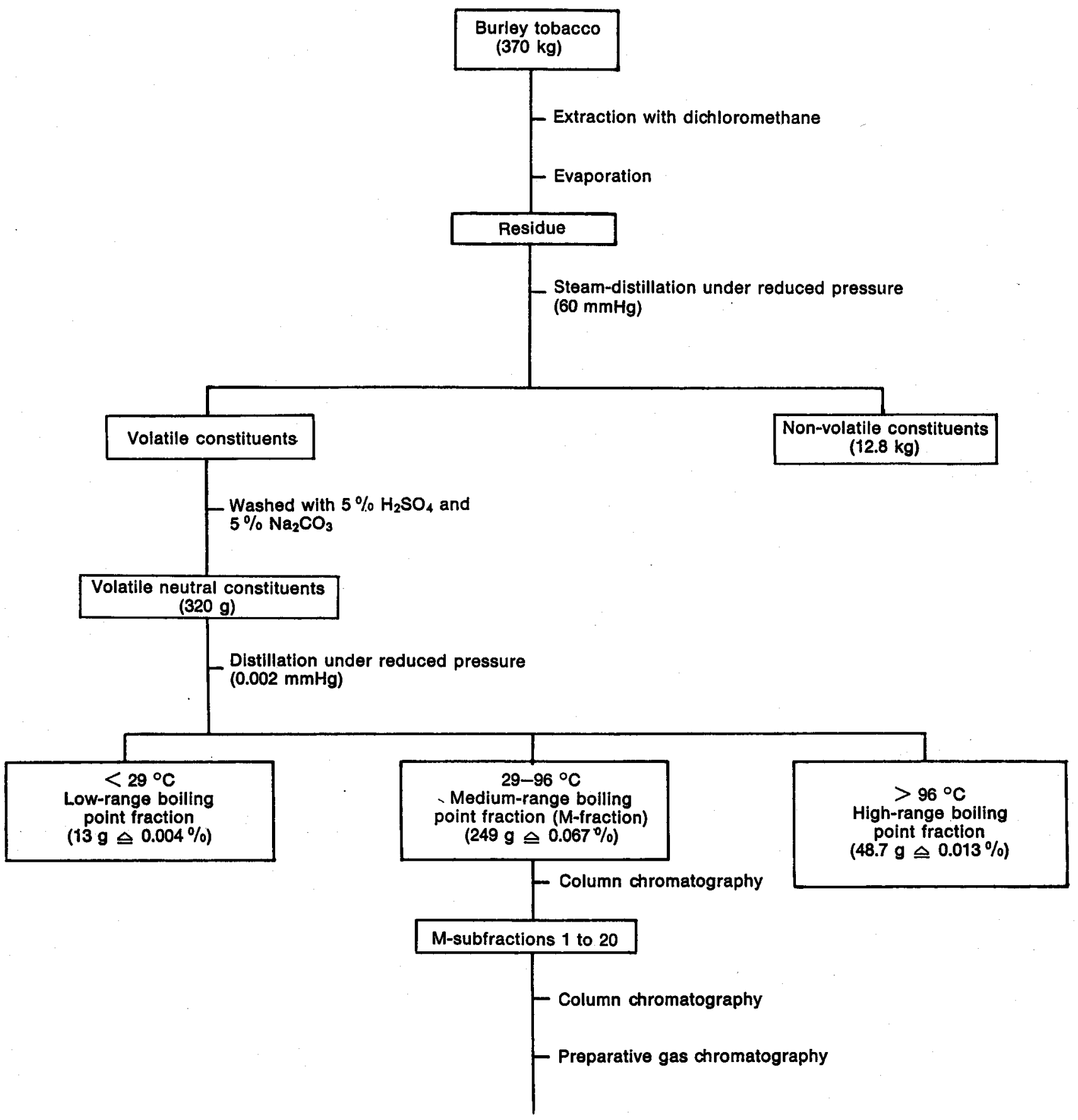


Flgure 3. Gas chromatogram of the neutral volattle fraction of alr-cured and aged leaves.

Column: $0.25 \mathrm{~mm} \times 60 \mathrm{~m}$, OV-101

Temperature: $100-200^{\circ} \mathrm{C}(2 \% / \mathrm{min})$

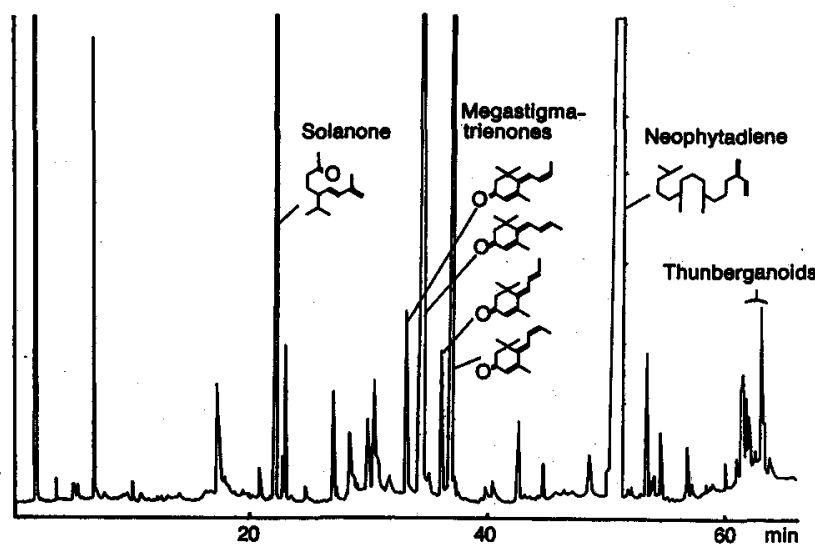

\section{RESULTS AND DISCUSSION}

A gas chromatogram of the neutral volatile fraction of air-cured and aged Burley tobacco using a $60 \mathrm{~m}$ glass capillary column coated with OV-101 is shown in Figure 3. The largest peak is neophytadiene, but it contributes little to tobacco flavor. Megastigmatrienones and solanone are representative aroma compounds in tobacco. The former are presumably derived from carotenoids, and the latter is from thunberganoids.

A gas chromatogram of the neutral volatile fraction prepared from Burley tobacco just before curing and aging is shown in Figure 4. Compared with the aged leaves, aroma components exist in a smaller amount. On the other hand, cyclic diterpenoids, thunberganoids, exist in a larger amount. These results suggest that most of the aroma components are generated during curing and aging.

\section{Compounds Obtained from Burley Tobacco Essential Oils}

The compounds identified (Table 1) include 12 hydrocarbons, 37 ketones and ketols, 6 esters, 22 alcohols and

Table 1. Classification of the Identified compounds in terms of functional groups.

\begin{tabular}{lc|c}
\cline { 2 - 3 } & $\begin{array}{c}\text { Number of } \\
\text { compounds }\end{array}$ & $\begin{array}{c}\% \text { in } \\
\text { M-frac- } \\
\text { tion* }\end{array}$ \\
\hline Hydrocarbons & 12 & 48.2 \\
Ketones and ketols & 37 & 11.9 \\
Esters & 6 & 3.3 \\
Alcohols and ethers & 22 & 2.8 \\
Lactones & 15 & 0.9 \\
Phenols and phenolic ethers & 4 & 0.4 \\
Furans & 2 & 0.1 \\
Aldehydes & 3 & trace \\
Nitrogenous compounds & 6 & trace
\end{tabular}

* medlum-range bolling point fraction
Figure 4. Gas chromatogram of the neutral volattle fraction of leaves before curing and aging.

Column: $0.25 \mathrm{~mm} \times 60 \mathrm{~m}, \mathrm{OV}-101$

Temperature: $100-200^{\circ} \mathrm{C}(2 \% / \mathrm{min})$

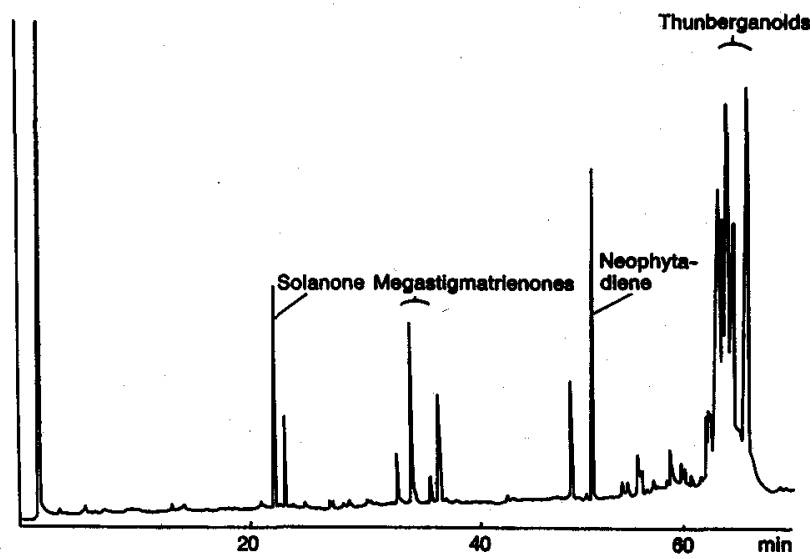

ethers, 15 lactones, 4 phenols and phenolic ethers, 2 furans, 3 aldehydes and 6 nitrogenous compounds $(1,2)$. The content of each group was evaluated by integration of the peaks in the gas chromatograms. Another classification of the identified compounds in terms of specific carbon skeletons is shown in Table 2. The 1st and 2nd groups contain carotenoid and thunberganoid-related compounds, respectively. These compounds had a char-

Table 2. Classification of the Identified compounds in terms of specific carbon skeletons.

\begin{tabular}{|c|c}
\hline $\begin{array}{c}\text { Num- } \\
\text { ber of }\end{array}$ & $\begin{array}{c}\% \text { in } \\
\text { com- }\end{array}$ \\
$\begin{array}{c}\text { com- } \\
\text { frac- } \\
\text { tion }\end{array}$ \\
\hline
\end{tabular}

1. Degradation products of carotenoids

25

7.7<smiles>CC=CC=C1C(C)=CC2CC(=O)CC1(C)C2</smiles><smiles>CC(=O)/C=C/C1=C(C)CC(O)CC1(C)C</smiles>

2. Degradation products of thunberganoids 14<smiles>C=C(C)/C=C/C(CCC(C)=O)C(C)C</smiles><smiles>CC(=O)C=CC(CCC(C)=O)C(C)C</smiles>

3. Neophytadiene-related compounds<smiles>C=CC(=C)CCC(C)(C)CCCC(C)CCCC(C)C</smiles><smiles>CC(C)(C)CCCC(C)(C)CCCc1ccoc1</smiles>

4. Mono and sesquiterpenoids<smiles>CC1=CCCC(C)=C1C</smiles><smiles>C=C1CC/C=C(/C)CCC2CC1(C)C2</smiles>

5. Bovolides
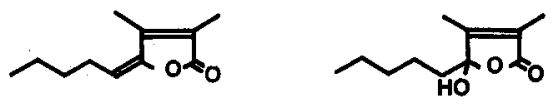

* medium-range bolling point fraction 
Figure 5. Thunberganold-related compounds.

THUNBERGANOIDS<smiles>C/C=C(/C)CCC(/C=C/C(C)(O)CC(O)/C=C(/C)CC/C=C(/C)CC)C(C)C</smiles><smiles>CC(=O)CCC(/C=C/C1(C)CCC(=O)O1)C(C)C</smiles>

10-0xo-4-methyl7-isopropylundec-5-en-4 olide<smiles>CC(=O)CCC(/C=C/C1(C)CCCO1)C(C)C</smiles>

5-Isopropyl-7(2-methyltetrahydrofur-2-yl)hept-6-en-2-one<smiles>CC(O)CCC(/C=C/C1(C)CCCO1)C(C)C</smiles>

5-Isopropyl-7(2-methyltetrahydrofur-2-yl)hept-6-en-2-ol<smiles>CC(O)C/C=C/C1(C)CCCO1</smiles>

6-Methyl-6,9oxidonon-4en-2-ol<smiles>CC(=O)C=CC(CCC(C)=O)C(C)C</smiles>

Norsolanadione<smiles>CC(=O)CCC(c1cc(C)co1)C(C)C</smiles>

Solanofuran

$$
\text { (17), 13- }
$$

duvatrien-1-ol<smiles>C=C(C)/C=C/C(CCC(C)=O)C(C)C</smiles>

Solanone<smiles>C=C(C)/C=C/C(CCC(C)O)C(C)C</smiles>

Solanol<smiles>CC(O)CCC(CCC(C)O)C(C)C</smiles>

5-Isopropylnonan-2,8-diol<smiles>CC(C)C1CCC(C)(C)OC1C(C)O</smiles>

3,3,5-Trimethyl8-isopropyl-4,9. dioxabicyclo[3.2.1]nonan-

$$
\text { 2-ol }
$$


Flgure 6. Photo-oxygenatlon of neophytadlene.<smiles>C=C(C)CCC(C)CCCC(C)CCCC(C)CCCC(C)CCCC(C)CCCc1ccco1</smiles>

E. Demole et al. (1973)

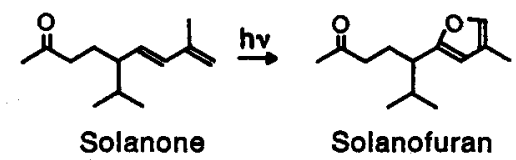

acteristic aroma and were thought to be key flavor components in the essential oils. The 3rd group contains neophytadiene-related compounds. Neophytadiene was present in a large quantity. It has been suggested that this compound originates from phytol (3). But evidence of this relation has not been confirmed experimentally. The 4th group contains mono and sesquiterpenoids. The 5th group contains bovolides which have a unique structure among the natural aroma constituents.
Figure 8. Solavetivone.

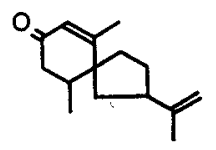

Solavetivone

R.C. Anderson et al. (1977)
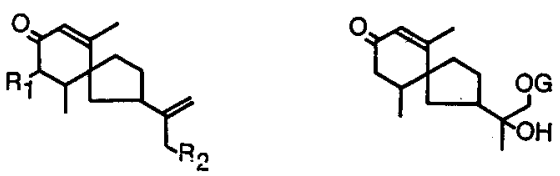

1. $R_{1}=0$-glycoside, $R_{2}=H$

2. $R_{1}=H_{1} R_{2}=0$-glycoside

Thunberganoid-Related Compounds: The compounds which seem to be degradation products of thunberganoids are organoleptically important (Fig. 5). Solanone and norsolanadione were obtained in large amounts. The occurrence of other diterpenoids, labdanoids, has been known in cigar tobacco and Oriental tobacco. In

\section{MONOTERPENOIDS}<smiles>C=CC(O)(CCC(C)C)CC(C)C</smiles>

Linalool<smiles>Cc1ccc(C(C)C)c(O)c1</smiles>

Thymol

Figure 7. Mono and sesquiterpenolds.<smiles>CCC=C(C)CCO</smiles>

Geraniol<smiles>CC1CCC(C(C)C)CC1</smiles>

1,8-Cineol<smiles>CC(C)=CCCC(C)CCO</smiles>

Citoronellol<smiles>C=C(C)C1CC=C(C)C(=O)C1</smiles>

Carvone<smiles>CC1=CCC(C(C)(C)O)CC1</smiles>

$\alpha-$ Terpineol

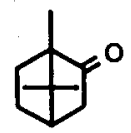

Camphor

\section{SESQUITERPENOIDS}<smiles>CC1(C)CCCC1(C)c1ccccc1</smiles>

Cupalene<smiles>C=CC1(C)CCC(C(=C)C)CC1C(=C)C</smiles>

$\beta$-Elemene<smiles>CC1=CCC(C(C)C)C2CC(C)CCC12O</smiles>

Cadinenol<smiles>Cc1ccc2c(c1)C(C(C)C)CCC2C</smiles>

Calamenene

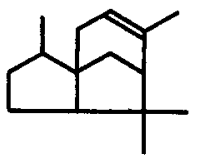

$\alpha$-Cedrene

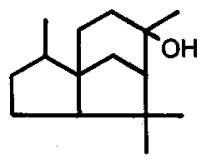

Cedrol<smiles>CC(C)=CCCC(C)c1ccc(C)cc1</smiles>

$\alpha$-Curcumene

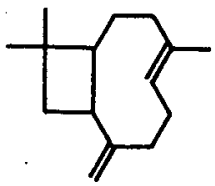

$\beta$-Caryophyllene<smiles>C=C(C)C1CCC2(C1)C(C)CC(=O)CC2C</smiles>

Solavetivone<smiles>CC1=CC2C(=C(C)CCC2C(C)C)CC1</smiles>

8-Cadinene

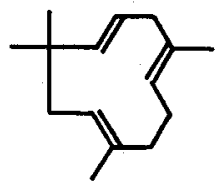

$\alpha$-Humulene<smiles>C=C(C)C1CCC2(C)CC(O)C(=O)C(C)=C2C1</smiles>

2-Keto- $\alpha$-cyperone 
Figure 10. Carotenoid-related compounds.

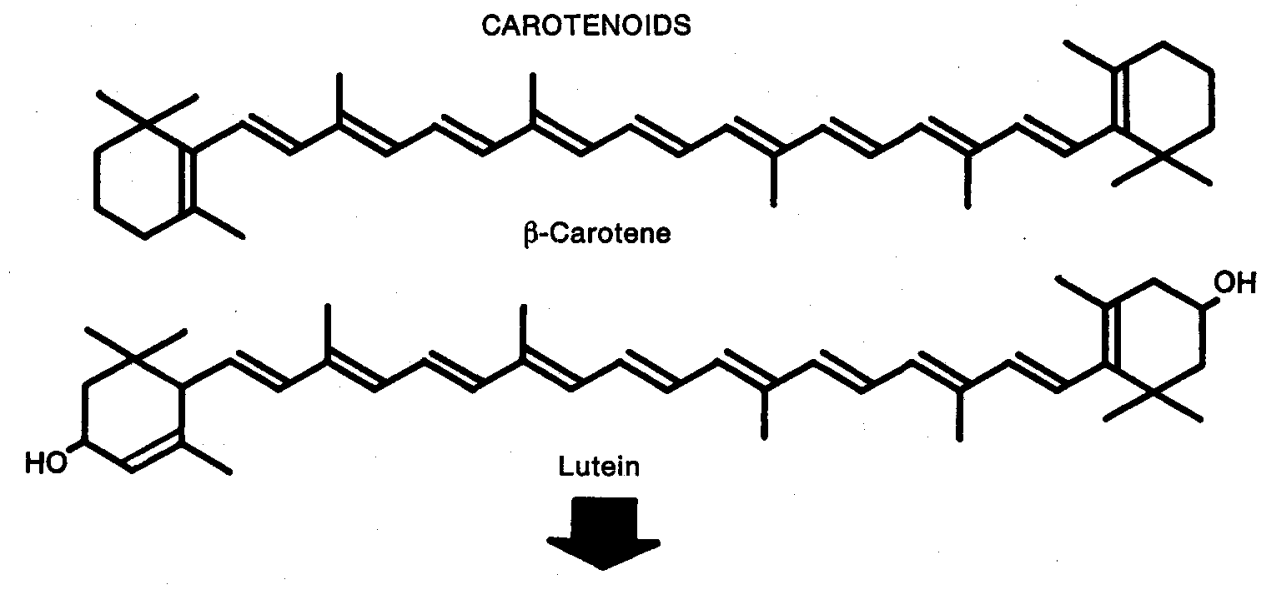

C9<smiles>CC1=CC(=O)CC(C)(C)C1</smiles>

Isophorone<smiles>CC1=CC(=O)CC(C)(C)C1=O</smiles>

2,6,6-Trimethylcyclohex-2-en-1,4-dione

$\mathrm{C}_{11}$<smiles>CC1(C)CCCC2(C)OC(=O)C=C12</smiles>

Dihydroactinidiolide<smiles>CC1(C)CC(=O)CC2(C)OC(=O)C=C12</smiles>

Dehydrololiolide<smiles>CC1CC(=O)CC(C)(C)C1O</smiles>

4-Hydroxy-3,3,5trimethylcyclohexanone<smiles>CC1(C)CC(=O)CC2(C)OCCC12</smiles>

1,5,5-Trimethyl-9-oxabicyclo[4.3.0]nonan3-one<smiles>CC1CC(O)CC(C)(C)C1=O</smiles>

4-Hydroxy-2,2,6-trimethylcyclohexanone<smiles>CC1(C)CC(O)CC2(C)OCCC12</smiles>

6-Hydroxy-4,4,7atrimethyloctahydrobenzofuran

$\mathrm{C}_{13}$<smiles>CC(=O)/C=C/C1C(C)=CCCC1(C)C</smiles>

$\alpha$-Ionone<smiles>C/C=C/C=C1C(C)=CC(=O)CC1(C)C</smiles>

Megastigmatrienone (5 stereo-isomers)<smiles>CC1=CC(=O)CC(C)(C)C1=CCC(C)O</smiles>

4-(3-Hydroxybutylidene)-3,5,5-trimethylcyclohex-2-en-1-one<smiles>CC(=O)/C=C/C1=C(C)CC(O)CC1(C)C</smiles>

3-Hydroxy- $\beta$-ionone<smiles>CC(=O)/C=C/C1=C(C)CCCC1(C)C</smiles>

$\beta$-lonone<smiles>C/C=C/C(=O)C1=C(C)CCCC1(C)C</smiles>

$\beta$-Damascone

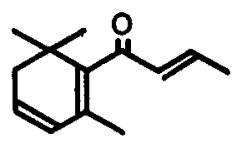

Damascenone<smiles>CC(=O)/C=C/c1c(C)ccc(C)c1C</smiles>

2,3,6-Trimethylbenzylidene acetone<smiles>CC1CCC2C(C)(C)CC(=O)CC2(C)O1</smiles>

1,3,7,7-Tetramethyl2-oxabicyclo[4.4.0]decan-9-one<smiles>CC1=CC(=O)CC(C)(C)C1CCC(C)O</smiles>

3-Oxo-7,8-dihydro$\alpha$-ionol<smiles>CC1=C(/C=C/C(C)O)C(C)(C)CC(O)C1</smiles>

3-Hydroxy- $\beta$-ionol<smiles>CC1CC=C2C(C)(C)CC(=O)CC2(C)O1</smiles>

1,3,7,7-Tetramethyl2-oxabicyclo[4.4.0]dec5-en-9-one<smiles>CC1=CC(=O)CC(C)(C)C1/C=C/C(C)O</smiles>

3-Oxo-a-ionol<smiles>CC(=O)/C=C/C1=C(C)C(=O)CCC1(C)C</smiles>

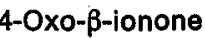<smiles>CC(O)C1C=C2C(C)(C)CC(=O)CC2(C)O1</smiles>

3-Oxoactinidol<smiles>C/C=C/C(=O)C1=C(C)CC(O)CC1(C)C</smiles><smiles>CC1=C(C#CC(C)O)C(C)(C)CC(O)C1</smiles>

3-Hydroxy-7,8-dehydro$\beta$-ionol
3-Hydroxy- $\beta$-damascone 
Flgure 9. Bovolides.

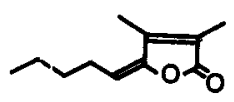

Bovolide

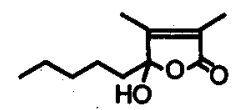

Hydroxydihydrobovolide

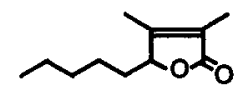

Dihydrobovolide<smiles></smiles>

Spiroxabovolide addition, many degradation products, such as norambreinolide, were reported from these tobaccos $(4,5)$. We could not recognize the presence of any labdanoid hydrocarbons or their oxygenated products in our Burley extract.

Phytofuran: Phytofuran was obtained as the first diterpenoid furan from tobacco extract (6). In advance of the isolation of this compound, solanofuran was isolated from Burley tobacco and prepared via the dye-sensitized photo-oxygenation of solanone by Demole et al. (7). Phytofuran was also prepared in a similar manner from neophytadiene (Fig. 6). These results may support the view that certain naturally, occurring terpenoid furans are formed from their conjugated diene precursors.

Mono and sesquiterpenoids: Eight monoterpenoids and 12 sesquiterpenoids were obtained (Fig. 7). In this group, solavetivone was a unique sesquiterpenoid which had a citrus-like fragrance (Fig. 8). This compound is the first component which has a vetispirane skeleton in tobacco (8). Solavetivone has been obtained as a major stress metabolite from potato tubers by Coxon et al. (9). Recently, sesquiterpene glucosides with a vetispirane skeleton were isolated from the water-soluble portion of an acetone extract of flue-cured tobacco by Anderson et al. (10).

Bovolides: Bovolides are unique in structure among natural substances (Fig. 9). Dihydrobovolide has been isolated from cigar tobacco as a celery-like odor compound (11). Hydroxydihydrobovolide was obtained (12). This compound also had a celery-like odor.

Carotenoid-Related Compounds: The compounds which seem to be degradation products of carotenoids are shown in Figur 10. These carotenoid-related compounds may be formed by oxidative cleavage of conjugated double bonds of carotenoids such as lutein (Fig. 11). Lutein and $\beta$-carotene are known to be predominant carotenoid components in tobacco leaves (13).

3 -Hydroxy-7,8-dehydro- $\beta$-ionol is the first acetylenic compound found in tobacco (14). It had a strong floral and tobacco-like fragrance. Damascenones and damascones are known as important aroma compounds in tobacco. Obloff et al. demonstrated that damascenone and 3 -hydroxy- $\beta$-damascone were converted from a tri or dihydroxyacetylenic compound by a synthetic experiment (15). The existence of 3 -hydroxy-7,8-dehydro- $\beta$-ionol suggests that Obloff's synthetic route type reaction may exist in tobacco (Fig. 12).

Figure 11. Plauslble route of the formation of the carotenold-related compounds.
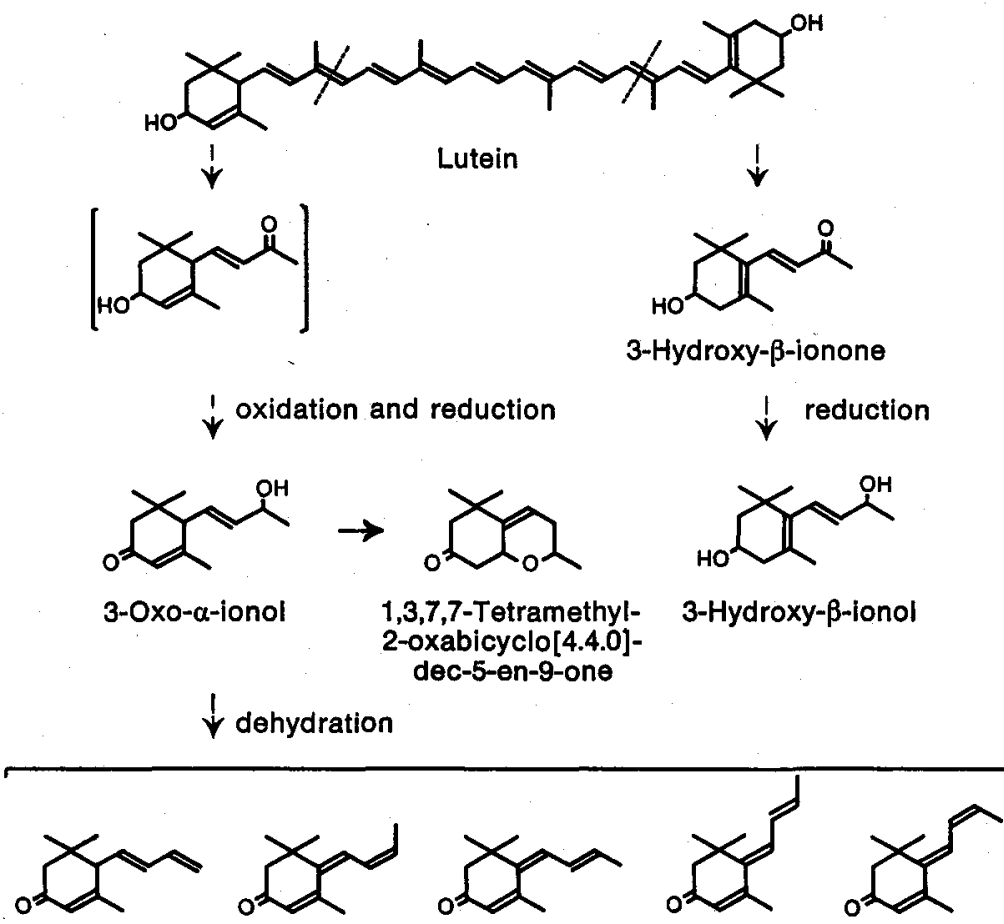

Megastigmatrienones (5 isomers) 
Figure 12. Possible reaction of 3-hydroxy-7,8-dehydro- $\beta$ Ionol (G. Ohloff et al., 1973).

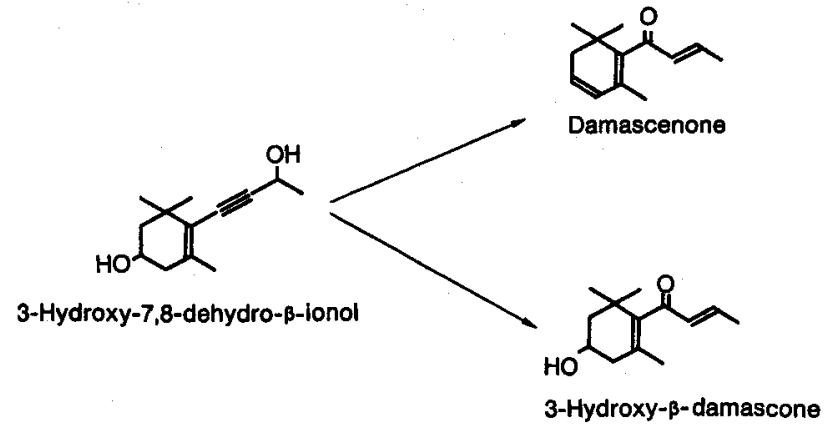

Dehydrololiolide had a hay-like fragrance (16). Recently, 3-oxoactinidol was obtained as a new tobacco constituent (16). 3-Oxoactinidol was a very unstable compound and was readily converted to dehydrololiolide at room temperature. The plausible mode of the formation of dehydrololiolide from lutein was shown in Figure 13.

\section{SUMMARY}

The constituents of the neutral volatiles in air-cured Burley tobacco were studied, and the following 19 substances were newly added to our lists:

1. 6-Methyl-6,9-oxidonon-4-en-2-ol

2. 5-Isopropylnon-3-en-2,8-diol

3. 5-Isopropylnonan-2,8-diol

4. 3,3,5-Trimethyl-8-isopropyl-4,9-dioxabicyclo[3.3.1] nonan-2-ol

5. 2-(1-Methyl-4-isopropyl-7,8-dioxabicyclo[3.2.1]octan-6-yl)propan-2-ol

6. 3-Oxoactinidol

7. 5-Isopropyl-7-(2-methyltetrahydrofur-2-yl)hept6-en-2-ol

8. o-Methyl acetophenone

9. 1,5,5-Trimethyl-9-oxabicyclo[4.3.0] nonan-3-one

10. 4-(3-Hydroxybutylidene)-3,5,5-trimethylcyclohex2-en-1-one

11. (1-Methyl-4-isopropyl-7,8-dioxabicyclo[3.2.1]octan-6-yl)methyl ketone

12. 6,10-Dimethyl-2-(1-methylethenyl)spiro[4.5]dec6-en-8-one (solavetivone)

13. 5-Isopropyl-7-(2-methyltetrahydrofur-2-yl)hept6-en-2-one

14. 4-Ethyl-4-methylbutan-4-olide

15. Phthalide

16. Hydroxydihydrobovolide

17. 1,2-Dimethoxy benzene

18. o-Anisidine

19. Methyl anthranilate

A large number of identified compounds may be viewed as degradation products of carotenoids and thunber-
Figure 13. Plausible route of the formation of dehydroIollollde.<smiles>CCC(C)(C)C=CC1=C(C)CC(O)CC1(C)C</smiles><smiles>CC(=O)/C=C/C1=C(C)CC(O)CC1(C)C</smiles>

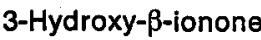<smiles>CC1=C(/C=C/C(C)O)C(C)(C)CC(O)C1</smiles>

3-Hydroxy- $\beta$-ionol í<smiles>CCC(O)/C=C/C1=C(C)CC(=O)CC1(C)C</smiles><smiles>CC1(C)CCCCC1(C)C=CC(O)CC1CCCCC1</smiles><smiles>CC(O)C1C=C2C(C)(C)CC(=O)CC2(C)O1</smiles>

3-Oxoactinidol<smiles>[Y][V]</smiles><smiles>CC1(C)CC(=O)CC1(C)OC(=O)C1CCCC1</smiles>

Dehydrololiolide ganoids. These compounds have a characteristic aroma and are thought to be key flavor components in the essential oils of tobacco. The presence of any labdanoid hydrocarbons or their oxygenated products was not recognized in our Burley tobacco extract. Burley tobacco is thought to be deficient in labdanoid compounds.

\section{ZUSAMMENFASSUNG}

Die Bestandteile der neutralen flüchtigen Fraktion von „air-cured“ Burleytabak wurden untersucht. Folgende 19 Substanzen konnten unserer Liste neu hinzugefügt werden:

1. 6-Methyl-6,9-oxidonon-4-en-2-ol

2. 5-Isopropylnon-3-en-2,8-diol

3. 5-Isopropylnonan-2,8-diol

4. 3,3,5-Trimethyl-8-isopropyl-4,9-dioxabicyclo[3.3.1]nonan-2-ol

5. 2-(1-Methyl-4-isopropyl-7,8-dioxabicyclo[3.2.1]octan-6-yl)-propan-2-ol

6. 3-Oxoactinidol

7. 5-Isopropyl-7-(2-methyltetrahydrofur-2-yl)-hept-6en-2-ol

8. o-Methylacetophenon

9. 1,5,5-Trimethyl-9-oxabicyclo[4.3.0]nonan-3-on

10. 4-(3-Hydroxybutyliden)-3,5,5-trimethylcyclohex-2en-1-on 
11. (1-Methyl-4-isopropyl-7,8-dioxabicyclo[3.2.1]octan6-yl)-methylketon

12. 6,10-Dimethyl-2-(1-methylethenyl)-spiro[4.5] dec-6en-8-on (Solavetivone)

13. 5-Isopropyl-7-(2-methyltetrahydrofur-2-yl)-hept-6en-2-on

14. 4-Ethyl-4-methylbutan-4-olid

15. Phthalid

16. Hydroxydihydro-bovolid

17. 1,2-Dimethoxy-benzol

18. o-Anisidin

19. Methylanthranilat

Eine große Zahl der identifizierten Verbindungen können als Abbauprodukte von Carotinoiden und Thunberganoiden angesehen werden. Sie besitzen ein dharakteristisches Aroma und scheinen die wesentlichen Vertreter der Geschmacksstoffe in den ätherischen Olen des Tabaks zu sein. Es konnten keine Labdan-Kohlenwasserstoffe oder deren oxigenierte Produkte im Extrakt des Burleytabaks nachgewiesen werden. Burleytabak scheint keine Labdanabkömmlinge $z u$ enthalten.

\section{RESUME}

On a étudié les constituants des volatiles neutres dans le tabac burley *air-cured $\star$. Les 19 substances suivantes ont pu être ajoutées à notre liste:

1. 6-Méthyl-6,9-oxidonon-4-ène-2-ol

2. 5-Isopropylnon-3-ène-2,8-diol

3. 5-Isopropylnonane-2,8-diol

4. 3,3,5-Triméthyl-8-isopropyl-4,9-dioxabicyclo[3.3.1]nonane-2-ol

5. 2-(1-Méthyl-4-isopropyl-7,8-dioxabicyclo[3.2.1]octane-6-yl)-propane-2-ol

6. 3-Oxoactinidol

7. 5-Isopropyl-7-(2-méthyltétrahydrofur-2-yl)-hept-6. ène-2-ol

8. o-Méthyl-acétophénone

9. 1,5,5-Triméthyl-9-oxabicyclo[4.3.0] nonane-3-one

10. 4-(3-Hydroxybutylidène)-3,5,5-triméthylcyclohex-2ène-1-one

11. (1-Méthyl-4-isopropyl-7,8-dioxabicyclo[3.2.1]octane-6-yl)-méthylcétone

12. 6,10-Diméthyl-2-(1-méthyléthényl)-spiro[4.5]dec-6ene-8-one (solavetivone)

13. 5-Isopropyl-7-(2-méthyltétrahydrofur-2-yl)-hept-6ène-2-one

14. 4-Éthyl-4-méthylbutane-4-olide

15. Phthalide

16. Hydroxydihydrobovolide

17. 1,2-Diméthoxy-benzène

18. o-Anisidine

19. Anthranilate de méthyle
Un grand nombre des composés identifiés peuvent être considérés comme produits de dégradation de caroténoïdes et de thunberganoïdes. Ces composés ont un arôme caractéristique et semblent être les principaux constituants odorants des huiles essentielles du tabac. On n'a pas constaté la présence d'hydrocarbures labdanoĩdes ou de leurs dérivés oxygénés dans notre extrait de tabac burley. Le tabac burley ne semble pas contenir de composés labdanoìdes.

\section{REFERENCES}

1. Fujimori, T., R. Kasuga, H. Matsushita, H. Kaneko and M. Noguchi: Agric. Biol. Chem. 40 (1976), 303.

2. Fujimori, T.: Scientific Papers of the Japan Tobacco and Salt Public Corporation, Central Research Institute, No. 118 (1976), 85 and Tob. Abstr. 21 (1977), 1823.

3. Rowland, R. L.: J. Amer. Chem. Soc. 79 (1957), 5007.

4. Kaneko, H.: Agric. Biol. Chem. 35 (1971), 1461.

5. Aasen, A. J., C. H. G. Vogt and C. R. Enzell: Acta Chem. Scand. B29 (1975), 51.

6. Fujimori, T., R. Kasuga, H. Kaneko and $M$. Noguchi: Agric. Biol. Chem. 38 (1974), 2293.

7. Demole, E., C. Demole and D. Berthet: Helv. Chim. Acta 56 (1973), 265.

8. Fujimori, T., R. Kasuga, H. Kaneko and M. Noguchi: Phytochem. 16 (1977), 392.

9. Coxon, D. T., K. R. Price, B. Howard, S. F. Osman, E. B. Kalan and R. M. Zacharius: Tetrahedron Letters 34 (1974), 2921.

10. Anderson, R. C., D. M. Gunn, J. Murray-Rust, P. Murray-Rust and J. S. Roberts: J. Chem. Soc. Chem. Commun. 1977, 27.

11. Kaneko, H., and M. Mita: Agric. Biol. Chem. 33 (1969), 1525.

12. Fujimori, T., R. Kasuga, H. Kaneko and M. Nogudi: in preparation.

13. Wright, H. E., Jr., W. W. Burton and R. C. Berry, Jr.: Arch. Biochem. Biophys. 82 (1959), 107.

14. Fujimori, T., R. Kasuga, H. Kaneko and M. Nogudi: Phytochem. 14 (1975), 2095.

15. a) Ohloff, G., D. Rautenstrauch and K. H. SchulteElte: Helv. Chim. Acta 56 (1973), 1503.

b) Ohloff, G.: 7th International Congress of Essential Oils, Kyoto, Japan, 1977.

16. Uegaki, R., T. Fujimori, H. Kaneko, K. Kato and M. Nogudi: Agric. Biol. Chem. 1979, in press.

\section{Authors' address:}

The Japan Tobacco and Salt Public Corporation, Central Research Institute, 6-2 Umegaoka, Midori-ku, Yokobama, Kanagawa, 227, Japan. 